\title{
ELECTROACTIVE POLYMERS AS ARTIFICIAL MUSCLES - CAPABILITIES, POTENTIALS AND CHALLENGES
}

\author{
Yoseph Bar-Cohen \\ JPL/Caltech, (MC 82-105), 4800 Oak Grove Drive, Pasadena, CA 91109-8099 \\ Phone 818-354-2610, Fax 818-393-4057, yosi@jpl.nasa.gov, web: http://ndea.jpl.nasa.gov
}

\section{INTRODUCTION}

The low density and the relative ease of shaping made polymers highly attractive materials and they are increasingly being chosen for aerospace applications. Polymer matrix composite materials significantly impacted the construction of high performance aircraft components and structures. In recent years, the resilience characteristics of polymers made them attractive to the emerging field of inflatable structures. Balloons were used to cushion the deployment of the Mars Pathfinder lander on July 4, 1997, paving the way for the recent large number of related initiatives. Inflatable structures are now being used to construct a rover (Figure 1), aerial vehicles, telescopes (Figure 2), radar antennas, and others. Some of these applications have reached space flight experiments, whereas others are now at advanced stages of development. Polymers were also reported as construction materials for such actuators as:

(a) McKibben muscle actuators [Schulte, 1961] - These are air tubes with an angularly breaded fiber reinforcement that contract significantly when inflated, delivering a large force.

(b) Shape memory polymers [Sokolowski, et al, 1999] - When subjected to pressure and heat, these materials sustain a volume reduction of over 40 times, and when subjected to an elevated temperature (about $60^{\circ} \mathrm{C}$ ) a recovery of the pre-pressed shape is obtained.

(c) Electrorhological fluids [Block and Kelly, 1988] -- These are electroactive polymer liquids that experience dramatic changes in rheological properties in the presence of an electric field.

The recent introduction of polymers that induce large displacements under electrical activation led to their consideration as potential actuators. The main attractive characteristic of Electroactive polymers (EAP) is their operational similarity to biological muscles, particularly their resilience and ability to induce large actuation strains. EAP were shown to enable unique robotic components and miniature devices. These materials can induce displacement as high as two orders of magnitude greater than the striction-limited, rigid and fragile electroactive ceramics (EAC). Also, they are superior to shape memory alloys (SMA) in their spectral response, lower density, and resilience. Generally, EAP are electrically hard and mechanically soft. Particularly, ferroelectric polymers have a coercive field in the range of $100 \mathrm{MV} / \mathrm{m}$, which is of the order of 100 times the coercive fields of ceramic ferroelectrics making polymers quite stable electrically. On the other hand, EAP materials reach their elastic limit at lower stress levels compared to EAC, and their actuation stress falls far shorter than EAC and SMA actuators. A comparison between EAP, EAC and SMA is given in Table 1 and it is easy to see the properties in which EAP offer superior characteristics.

The most attractive feature of EAPs is their ability to emulate biological muscles offering high toughness, large actuation strain and inherent vibration damping. This similarity gained them the name "Artificial Muscles" with the potential of developing biologically inspired robots.

Biomimetic robots actuated by EAP can be made highly maneuverable, noiseless and agile, with various shapes. Effective EAP allow making science fiction ideas a faster reality than would be feasible with any other conventional actuation mechanisms. Unfortunately, the force actuation and mechanical energy density of EAPs are relatively low, limiting the potential applications that can be considered at the present time. In recognition of the need for international cooperation among the developers, users and potential sponsors, the author 
organized though SPIE International the first EAP Conference on March 1-2, 1999. This Conference was held in Newport Beach, California, USA and was the largest ever on this subject, making an important milestone, and turning the spotlight onto these emerging materials and their potential. Following this success, MRS conference was initiated to address the fundamental issues related to the material science of EAP. Further, the author established a homepage linking websites of worldwide EAP research and development facilities (http://ndeaa.jpl.nasa.gov/nasa-nde/lommas/eap/EAP-web.htm). Also, he initiated the publication of the WW-EAP Newsletter, which is published electronic (http:/eis.jpl.nasa.gov/ndeaa/nasande/newsltr/WW-EAP_Newsletter.PDF) and help fostering the WW-EAP Newsgroup.

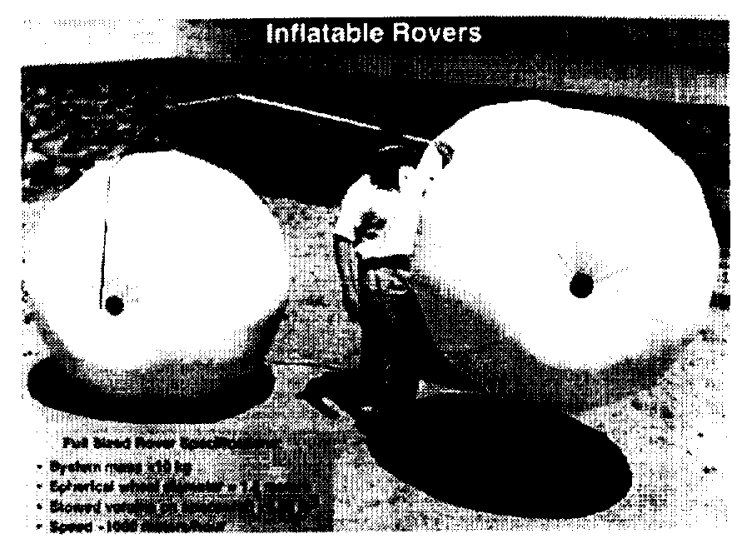

FIGURE 1: JPL rover using inflatable wheels.

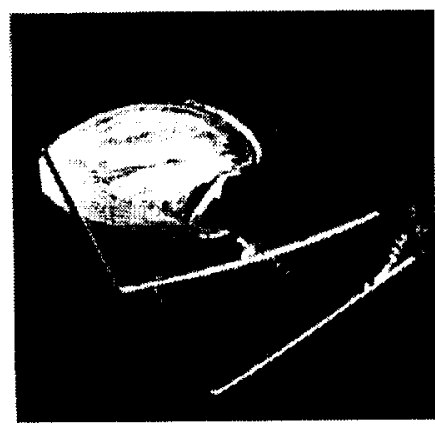

FIGURE 2: A Space Shuttle view of an inflatable structure experiment (May 1996). a. During inflation; and $b$. Fully open.

TABLE 1: Comparison of the properties of some actuation materials

\begin{tabular}{|l|c|c|c|c|c|}
\hline \multicolumn{1}{|c|}{ Property } & $\begin{array}{c}\text { Electro-static } \\
\text { silicone } \\
\text { elastomer } \\
{[\text { Perline] }}\end{array}$ & $\begin{array}{c}\text { Polymer } \\
\text { Electrostrictor } \\
\text { [Zhang] }\end{array}$ & SMA & $\begin{array}{c}\text { Single Crystal } \\
\text { Electrostrictor } \\
\text { [Shrout] }\end{array}$ & $\begin{array}{c}\text { Single Crystal } \\
\text { Magnetostrictor } \\
\text { [Clark] }\end{array}$ \\
\hline Actuation strain & $32 \%$ & $4 \%$ & $8 \%$ & $1.7 \%$ & $2 \%$ \\
\hline Blocking Force/Area & $0.2 \mathrm{MPa}$ & $0.8 \mathrm{MPa}$ & $700 \mathrm{MPa}$ & $65 \mathrm{MPa}$ & $100 \mathrm{MPa}$ \\
\hline Reaction speed & $\mu \mathrm{sec}$ & $\mu \mathrm{sec}$ & sec to min & $\mu \mathrm{sec}$ & $\mu \mathrm{sec}$ \\
\hline Density & $1.5 \mathrm{~g} / \mathrm{cc}$ & $3 \mathrm{~g} / \mathrm{cc}$ & $6 \mathrm{~g} / \mathrm{cc}$ & $7.5 \mathrm{~g} / \mathrm{cc}$ & $9.2 \mathrm{~g} / \mathrm{cc}$ \\
\hline Drive field & $144 \mathrm{~V} / \mu \mathrm{m}$ & $150 \mathrm{~V} / \mu \mathrm{m}$ & -- & $12 \mathrm{~V} / \mu \mathrm{m}$ & $2500 \mathrm{Oe}$ \\
\hline Fracture toughness & large & large & large & low & large \\
\hline
\end{tabular}

Note: Values were calculated assuming the elastic properties were independent of applied field and are therefore approximated.

The increased resources, the grows in number of investigators that are conducting EAP related research, and the improved collaboration among the developers, users and sponsors are all expected to lead to rapid progress in the coming years. Recently, the author challenged the worldwide community of EAP experts to develop a robotic arm that is actuated by artificial muscles to win a wrestling match with a human opponent (Figure 3). Progress towards this goal will lead

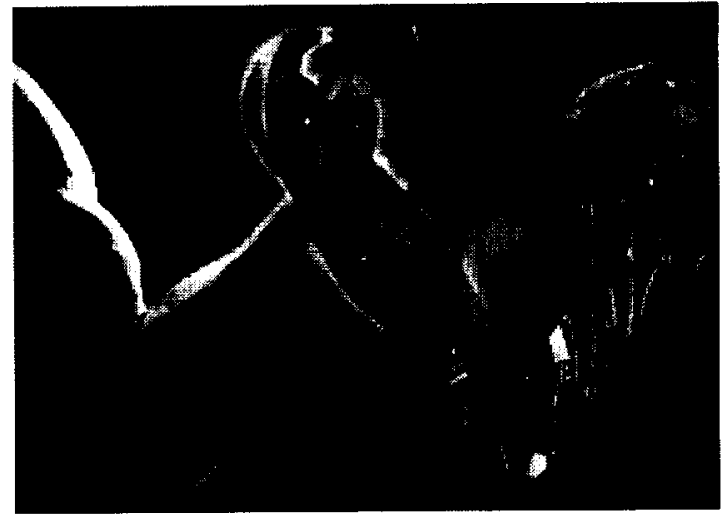

FIGURE 3: Grand challenge for the EAP community. 
to great benefits, particularly in the medical area, including effective prosthetics. Decades from now, EAP may be used to replace damaged human muscles, leading to a "bionic human." A remarkable contribution of the EAP field would be to one day seeing a handicapped person jogging to the grocery store using this technology.

\section{NEED FOR AN EFFECTIVE EAP INFRASTRUCTURE}

Construction of a mobile or an articulation system that is actuated by EAP requires components as shown in the block diagram of Figure 4 . While each of the listed components is at various advanced research phases, EAP actuators are the least developed technology and extensive effort is required to bring it to a mature stage. Unfortunately, the materials that have emerged so far are still exhibiting low force and are far from being effective. Moreover, there are no commercially available robust EAP materials that can be considered for application in practical devices. In recent years, a series of EAP materials that induce large displacements were documented, including ion exchange membranes, carbon nanotubes, conductive polymers, electrostrictives, electrostatics and piezoelectrics [Bar-Cohen, 1999a]. In order to be able to transition these materials from a development phase to effective actuators

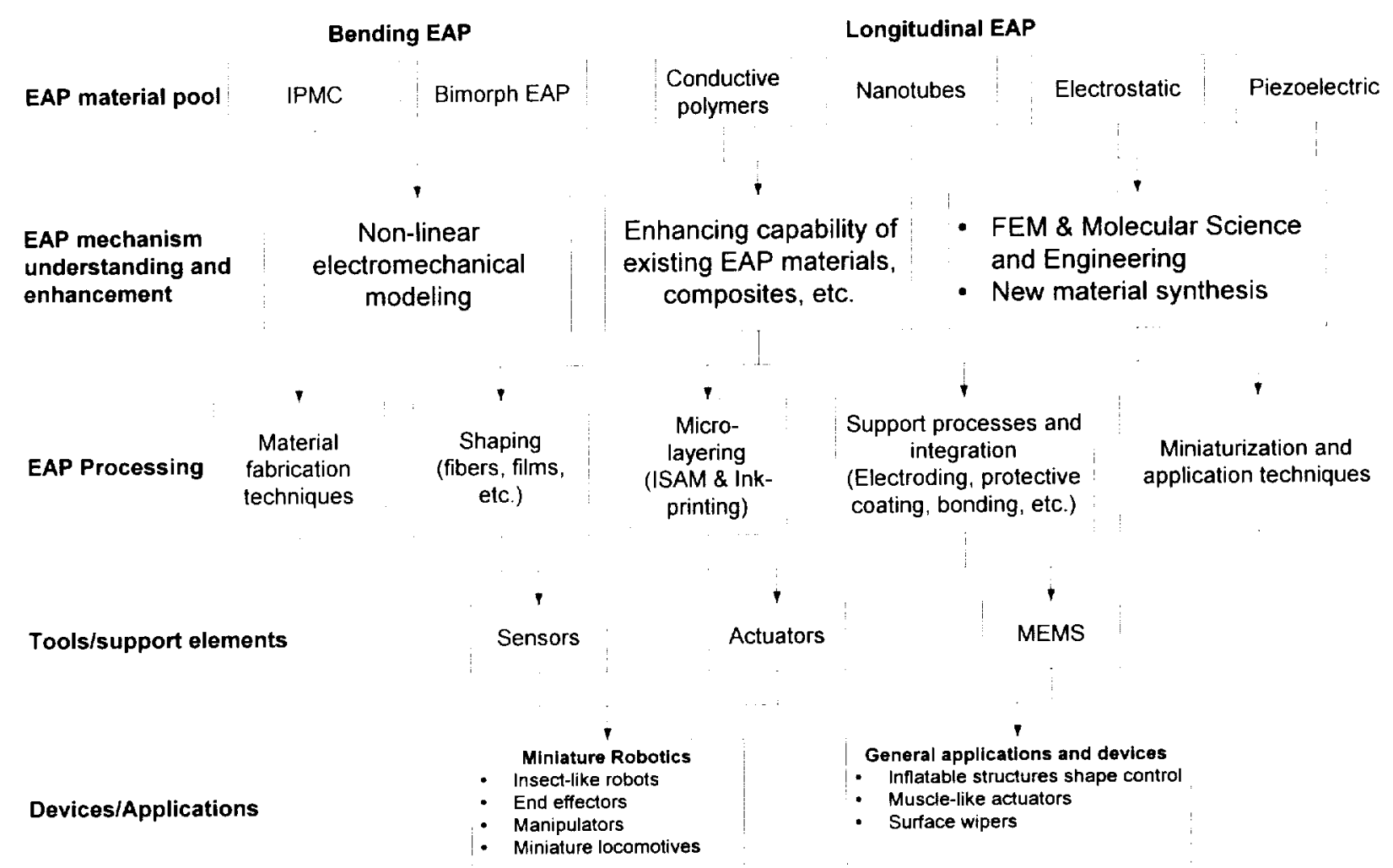

FIGURE 5: EAP infrastructure and areas needing attention. 
there is a need to establish an adequate "EAP infrastructure". The author's view of this infrastructure and the areas needing simultaneous development are shown schematically in Figure 5. This involves the need for adequate understanding of EAP materials' behavior and the requirements necessary to assure their durability under various service conditions. Further, enhancing their actuation force will require understanding the basic principles, development of computational chemistry models, comprehensive material science, electro-mechanic analytical tools and improved materials processes. Efforts are needed to gain a better understanding of the parameters that control the EAP electro-activation force and deformation. The processes of synthesizing, fabricating, electroding, shaping and handling will need to be refined to maximize their actuation capability and robustness. Methods of reliably characterizing the response of these materials are required to allow documenting the material properties to support design engineering and making EAP the actuators of choice. Various configurations of EAP actuators, sensors and potential MEMS will need to be studied and modeled to produce an arsenal of effective actuators. The development of the infrastructure is multidisciplinary and requires international collaboration.

\section{BIOLOGICAL MUSCLES AND SCIENCE FICTION}

Development of intelligent robots requires the combination of strong muscles (actuators) and acute sensors, as well as the understanding of the biological model. Using effective EAP materials, as artificial muscles, one can develop biologically inspired robots and locomotives that can possibly walk, fly, hop, dig, swim and/or dive. Natural muscles are driven by a complex mechanism and are capable of lifting large loads at short (millisecond) response times. The performance characteristics of muscles are difficult to measure and most measurements were made on large shell-closing muscles of scallops. Peak stress of $150-300-\mathrm{KPa}$ is developed at a strain of about $25 \%$. Maximum power output is $150-225-\mathrm{W} / \mathrm{kg}$; average power is about $50-$ $\mathrm{W} / \mathrm{kg}$ with an energy density of $20-70-\mathrm{J} / \mathrm{kg}$, which decreases with the increase in speed. Since muscle is fundamental to animal life and changes little between species, we can regard it as a highly optimized system. It is system that depends on chemically driven reversible hydrogen bonding between two polymers, actin and myosin. Muscle cells are roughly cylindrical, with diameters between 10 and $100-\mu \mathrm{m}$ and up to few centimeters long. Although muscles produce linear forces, motions at joints are all rotary. Therefore, the strength of an animal is not just muscle force, but muscle force as modified by the mechanical advantage of the joint [Alexander, 1988], which usually varies with joint rotation (as does the muscle force). The mechanical energy is provided by a chemical free energy of a reaction involving adenosine triphosphate (ATP) hydrolysis. The release of $\mathrm{Ca}+2$ ions seems turning on and off the conformational changes associated with muscle striction.

Insects mobility is under extensive study and there is a relatively large body of knowledge in place, as for example at the University of California, Berkeley [Full and Tu, 1990]. A windmill was used with a photoelastic coating to study the details of insects walking mechanisms, where insects with various numbers of legs were investigated. Also, the size of electronic devices has become so small that insects can be instrumented to perform tasks once viewed as science fiction. At the University of Tokyo, Japan, a spider and other insects were instrumented as locomotives to carry backpacks of wireless electronics. Development in EAP actuators is expected to enable insect-like robots that can be launched into hidden areas of structures (e.g., aircraft engine) to perform inspection and various maintenance tasks. In future years, EAP may emulate the capabilities of terrestrial creatures with integrated multidisciplinary capabilities to launch missions with innovative plots. Some biological functions that can be adapted include soft-landing like cats, traversing distances by hopping like a grasshopper and digging and operating cooperatively as ants. 


\section{CURRENTLY AVAILABLE EAP MATERIALS}

The beginning of the field of electroactive polymers can be traced back to the milestone discovery of an electret when carnauba wax, rosin and beeswax are solidified by cooling while subjected to DC bias field [Eguchi, 1925]. Generally, electrical excitation in only one of the mechanisms of inducing elastic deformation in polymers [Perline, et al, 1998, and Zhang, et al, 1998]. Other activation mechanisms include chemical [Kuhn, et al, 1950; Steinberg, et al, 1966; and Otero, et al, 1995], thermal [Tobushi, et al, 1992; and Li, et al, 1999], magnetic [Zrinyi, et al, 1997], and optical [van der Veen \& Prins, 1971]. Polymers that are chemically stimulated were discovered before half a century when collagen filaments were demonstrated to reversibly contract or expand when dipped in acid or alkali aqueous solutions, respectively. Even though very little has since been done to exploit such 'chemo-mechanical' actuators [Sussman, 1975], this early work pioneered the development of synthetic polymers that mimic biological muscles. The convenience and the practicality of electrical stimulation as well as technical progress led to a continuously growing emphasis on electroactive polymer materials. Following the 1969 observation of a substantial piezoelectric activity in PVF2, investigators started to examine other polymer systems and a series of effective materials have emerged. While the list of such polymers has grown considerably, PVF2-TrFE is the only EAP material that can be obtained commercially. The emerging EAP can be divided into two categories: Wet (ionic) and dry polymer materials. Generally, the dry material (electrostrictive, electrostatic, piezoelectric and ferroelectric) require high activation voltage $(>100-\mathrm{V} / \mu \mathrm{m})$ that is close to the breakdown level. However, the material can be made to hold its displacement under DC voltage allowing considerations for robotic applications. Also, these materials have a greater mechanical energy density. On the other hand, the wet (ionic) EAP (Ion-exchange, conductive polymers, gels, etc.) require several volts, which can be as low as 1-2 Volts. Their drawbacks are the need to maintain wetness and the difficulty to sustain the induced displacement under an actuation DC voltage. Some of these EAP materials naturally bend, whereas others become longer or shorter depending on the polarity orientation of the activation field and the material characteristics. Generally, any of the EAP can be made to produce a bending actuator with a significant bending response, which appears appealing. However, such actuators have relatively limited applications due to the limited drive force that can be induced.

\section{WET (IONIC) EAP}

\section{IONIC CONDUCTIVE POLYMERS (ICP)}

Ionic conductive polymers [Otero, et al, 1995, and Tanaka, et al, 1982] are materials containing solvated ions that cause swelling in response to an applied voltage. Electrophoresis or electroosmotic drag mechanisms induce conformational changes. Swelling of the polymer can occur even in the absence of applied fields as a result of sorbtion of solvents (usually water). Electrochemical reactions (oxidation/reduction) occur at the electrodes that either promote or hinder the actuation mechanism. Most reported actuators that were formed using ICP materials exploited the voltage controlled swelling to form bending actuators. The required voltage may vary from $1-\mathrm{mV}$ to $50-\mathrm{V}$ and the response time depends on thickness, diffusivity, and kinetics of electrochemical reactions. The reaction rate of these types of EAP materials can vary from milliseconds to minutes. Protective coating, that act as artificial skin, needs to be developed in order to practically operate these materials in dry environments. The use of polar low-vaporpressure solvents (such as propylene carbonate) can help extending the ability of ICP to operate at harsh conditions. Actuators that are based on ICP are inducing relatively low actuation force, which are typically less than 1 -g. In recent years, several conductive polymers were reported including: Polypyrrole, Polyethylenedioxythiophene, Poly(p-phenylene vinylene)s, Polyaniline (PANi), and Polythiophenes. 


\section{IONIC GELS}

Ionic gels can be synthesized to produce actuators having the potential of matching the force and energy density of biological muscles. These materials are generally activated by a chemical reaction, where changing from an acid to base environment cause the gel to become dense or swollen, respectively. This reaction can be simulated electrically as shown by researchers at the University of Arizona, USA [Calvert, et al, 1998]. When driven by embedded electrodes, these gels bend as the cathode side becomes more basic and the anode side more acidic. However, the response of this multilayered gel structure is relatively slow because of the need to diffuse ions into the gel. Expanding and shrinking from $3 \times 3-\mathrm{cm}$ to $6 \times 6-\mathrm{cm}$ occurred over a period of 20 min, which is far from being practical. Also, the significant displacement is causing damage to the electrode leading to a complete failure of the actuator after 2-3 three cycles of activation. Current efforts are directed towards the use of thin layers and more robust electroding techniques.

\section{IONIC POLYMER METALLIC COMPOSITE (IPMC)}

Perfluorinated ion exchange membrane platinum composite (IMPC) with metallic electrodes deposited on both sides is a bending EAP material. Two types of base polymers are used to form IPMC: Nafion ${ }^{\circledR}$ (perfluorosulfonate made by DuPont) and Flemion ${ }^{\circledR}$ (perfluorocaboxylate, made by Asahi Glass, Japan).

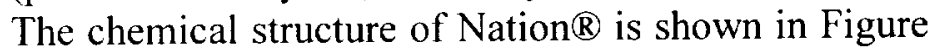
6. Prior to using these polymers as EAP base

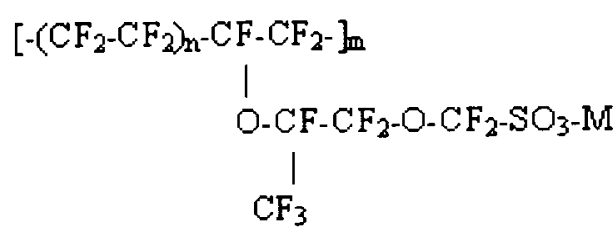

FIGURE 6: Nafion@ chemical structure (Where $\mathrm{n} \sim 6.5,100<\mathrm{m}<1000$, and $\mathrm{M}^{+}$ is the counter ion $\left(\mathrm{H}^{+}, \mathrm{Li}^{+}, \mathrm{Na}^{+}\right.$or others). materials, they were widely employed in fuel cells and production of hydrogen (hydrolysis). The operation as actuators is the reverse process of the charge storage mechanism associated with fuel cells. A relatively low voltage is required to stimulate bending in IPMC, where the base polymer provides channels for mobility of positive ions in a fixed network of negative ions. Since the author has been involved extensively with research related to this type of EAP, a greater attention will be given herein to this family of materials [Bar-Cohen, et al, 1998]. IPMC, as an EAP material, was realized in 1992 by [Oguro, et al, 1992] in Japan, and by [Sadeghipour, et al, 1992] and [Shahinpoor, 1992] in the United States. In order to chemically electrode IPMC films, metal ions (Platinum, gold or others) are dispersed throughout the hydrophilic regions of the polymer, and are subsequently reduced to the corresponding zero-valence metal atoms. Generally, the ionic content of the IPMC is an important factor in the electromechanical response of these materials. Displacement results for sodium cations and platinum metallization are shown in Figures 7 [Bar-Cohen, et al, 1998] showing large bending at low frequencies. In recent years, this bending capability was significantly enhanced using $\mathrm{Li}+$, tetra-n-butylammonium and other types of cations using gold metallization [Yoshiko, et al, 1998 and Oguro, et al, 1999].

The structure and properties of the IMPC have

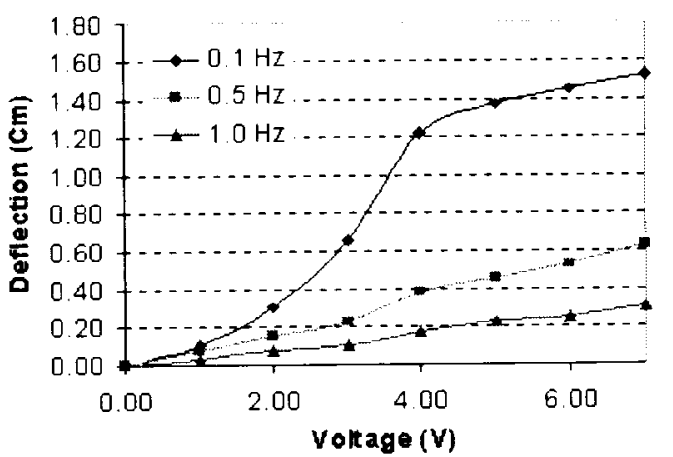

Figure 7: Typical response of $\mathrm{Na}+/ \mathrm{Pt}$ IPMC at various voltaae levels and 3 freauencies been the subject of numerous investigations (see for example [Heitner-Wirguin, 1996]). While its operation mechanism is still not clear, recent progress has been reported with regards to the methodic modeling of the material behavior. Diffusion and coulomb forces are considered as the driving mechanisms [Nemat-Naser et al, 2000]. One of the interesting properties of this material is its ability to absorb large amounts of polar solvents, i.e. water. When equilibrated with aqueous solutions, the polymer membrane swells to absorb certain amount of water. 
Swelling equilibrium results from the balance between the elastic forces of the polymeric matrix and the water affinity to the fixed ion-exchanging sites and the moving counter ions. The mechanism of bending is partially related to migration of mobile ions within the networks caused by an applied electric field. The structure and properties of IMPCs have been the subject of numerous investigations (e.g., [Heitner-Wirguin, 1996]). Recent investigation by [Firoozbakhsh \& Shahinpoor, 1998; and Nemat-Naser et al, 2000], suggests a strong interaction effect of surface charges.

As an actuator activated by 1 to 10 Volts, IPMC can create a large bending displacement when using low frequencies (see Figure 7) in the range of fractions of Hertz. Under a DC activation, the material bends relatively quickly (depending on the size of the cations, the rate can be 0.1 to 1 -second) and a slow recoiling occurs with a permanent deformation. The recoiling can be as serious as bending in the opposite direction of the activation voltage, as in the case of $\mathrm{Na}+$ cations. IPMC was also found to respond at temperatures as low as $-100^{\circ} \mathrm{C}$ where an increase in voltage allows compensating for the loss in efficiency. This result pave the path for considering IMPC as an actuator for space mechanisms as will be discussed later in this manuscript.

The author and his colleagues investigated the issues that affect the application of IPMC as an actuator and identified a series of challenges. The challenges and the solutions that were determined are listed in Table 2. As can be seen from the table most of the challenges where met with an acceptable solution except for three: the need to protect the material from drying, the excitation of electrolysis at voltages above 1.03 Volts and the induced permanent deformation. Unless these issues are effectively addressed the use of IPMC for planetary applications will be hampered.

TABLE 2: Challenges and identified solutions for issues regarding the application of IPMC.

\begin{tabular}{|l|l|}
\hline \multicolumn{1}{|c|}{ Challenge } & \multicolumn{1}{c|}{ Solution } \\
\hline Fluorinate base - difficult to bond & Apply pre-etching \\
\hline Sensitive to dehydration & Apply protective coating over pre-etched IPMC \\
\hline Off-axis bending actuation & Constrain the free end \\
\hline Most bending occurs near the poles & Improve the metal layer uniformity \\
\hline Electrolysis occurs at $>1.03-V$ & Use efficient IPMC requiring lower actuation voltage \\
\hline Operate at low temperatures & IPMC was demonstrated to operate at $-140^{\circ} \mathrm{C}$ \\
\hline Remove small size dust & Use effective wiper-blade design and high bias voltage \\
\hline Reverse bending under DC voltage & Limit application to dynamic operations \\
\hline Protective coating is permeable & Develop alternative coating possibly using overcoat \\
\hline Residual deformation & Still a challenge \\
\hline No established quality assurance & Use short beam/film and tackle the critical issues \\
\hline
\end{tabular}

Ineffective protective coating: Since the actuation capability of IPMC is attributed to its ionic content, it is necessary to continuously maintain its moisture. A protective coating was developed to serve as the equivalence of a biological skin, and was demonstrated to effectively maintain the moisture for several months [Bar-Cohen, et al, 1998]. Unfortunately, this silicone base coating was found to be too permeable and therefore would not be effective for long term operation of the IPMC in dry conditions. Efforts are underway to identify a better coating technique.

Electrolysis: The wetness of IPMC and the introduction of voltages at levels above $1.03-\mathrm{V}$ introduce electrolysis during electro-activation causing degradation, heat and release of gasses. This issue raises a great concern since the emitted hydrogen accumulates under the protective coating and leads to blistering, which will rupture the coating due to the high vacuum environment of space. The use of tetra-n-butylammonium cations was shown to provide higher 
actuation efficiency allowing to reduce the needed voltage and to minimizing the electrolysis effect. However, large bending requires voltages at significantly higher that the $1.03-\mathrm{V}$ limit.

Permanent deformation under DC activation: Unfortunately, under DC voltage IPMC strips do not maintain the actuation displacement and they retract after several seconds. Further, upon removal of the electric field an overshoot displacement occurs in the opposite direction moving slowly towards the steady state position leaving a permanent deformation. This issue was not resolved yet and would hamper the application of IPMC.

\section{Carbon Nanotubes}

Nanotubes are relatively new EAP materials, which have immerged in 1999 [Baughman, 1999]. They are composed of carbon nanotubes having diamond-like mechanical properties. Considering the mechanical strength and modulus of the individual carbon nanotubes as well as the achievable actuator displacements, the new actuators have the potential of providing higher work per cycle than previous actuator technologies and of generating much higher mechanical stresses. Further, since carbon offers high thermal stability, carbon nanotubes may eventually be used at temperatures exceeding $1000^{\circ} \mathrm{C}$, which far exceeds the capabilities of alternative highperformance actuator materials. The material consists of nanometer size tubes and it was shown to induce stains at the range of $1 \%$ along the length. The key obstacle to their commercialization is their high cost and the difficulty to mass-produce the material. A carbon nanotube actuator can be constructed in about ten minutes by simply laminating together two narrow strips cut from a carbon nanotube sheet, using an intermediate adhesive layer which is electronically insulating. The resulting "cantilever device" is immersed in an electrolyte, such as a sodium chloride solution, and an electrical connection is made to the two nanotube strips. The application of about a volt bends the actuator in one direction, and reversing the potential bends the actuator in the opposite direction.

\section{$\underline{D R Y E A P}$}

\section{ELECTRO-STATICALLY STRICTED POLYMER (ESSP) ACTUATORS}

Polymers with low elastic stiffness and high dielectric constant can be used to induce large actuation strain by subjecting them to an electrostatic field. This characteristic allows producing longitudinal actuators that operate similarly to biological muscles using Coulomb forces between electrodes to squeeze or stretch the material. Longitudinal electrostatic actuators can be made of a dielectric elastomer films and flexible electrodes [Perline, et al, 1998]. For this purpose two silicone layers can be used with carbon electrodes on both sides of one of the

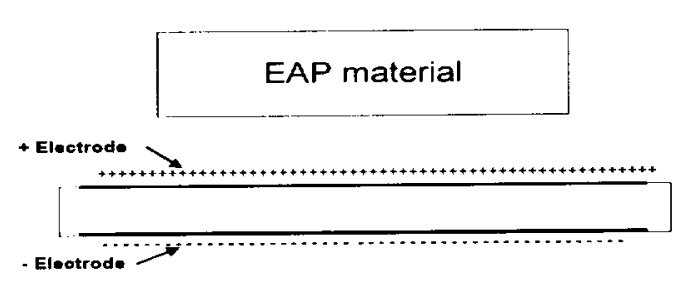

Figure 9: Left - Under electro-activation, an EAP film with electrodes on both surfaces expands laterally. Middle - EAP film subjected to $25 \mathrm{~V} / \mu \mathrm{m}$ induced over $12 \%$ extension. Right $-A>10-g$ rock mounted at the end of an EAP rope is lifted and dropped electrically.

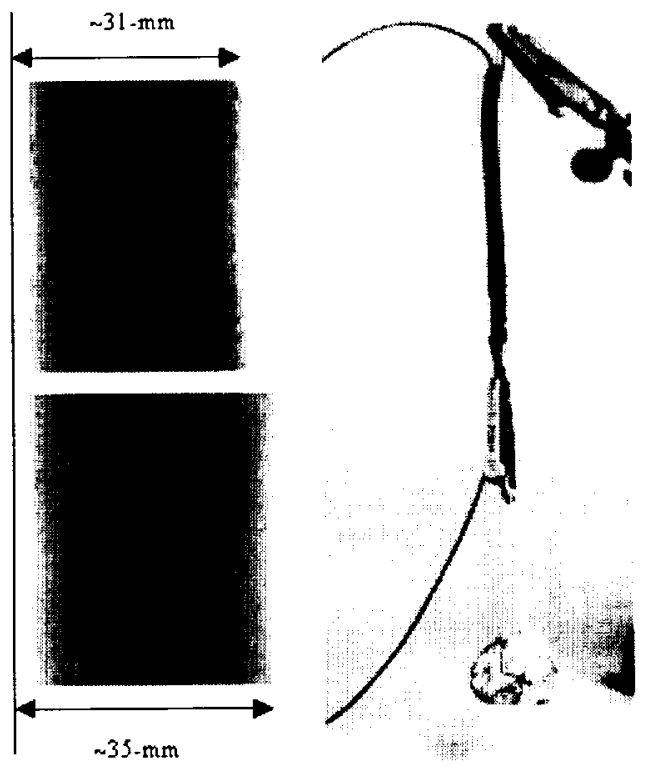


layers, where the layers are wrapped to form a rope as shown in Figure 8. Besides using ESSP in the form of ropes that are bundled to further mimic human muscle, bending actuators can be constructed by adding a passive backing layer on one side of the electroactive film.

ESSP actuators are subject to a major concern associated with the required large electric fields $(\sim 100 \mathrm{~V} / \mu \mathrm{m})$ necessary to induce significant strains $(10-200 \%)$. The actuator has to be thin $(<50 \mu \mathrm{m})$ to assure the use of achieve reasonable voltages. Overall, the associated voltage levels are close to the breakdown strength of the material and a safety factor needs to be used when designing such actuators thus lowering its potential. Moreover, the relatively small breakdown strength of air $(2-3 \mathrm{~V} / \mu \mathrm{m})$ presents additional challenge. Longitudinal actuators are produced using ESSP materials and they employ the Poisson effect that results from the film contraction. To obtain significant actuation displacements requires the use of large film material. Elastomers with Young's moduli on the order of $<20 \mathrm{MPa}$ and relative permitivities of 3 can induce large strain at the level of $30 \%$. The Young's modulus is fairly temperature independent until the glass transition temperature is reached, at which point a sharp increase occurs making them too stiff to be used as electrostatic actuators.

\section{FERROELECTRIC POLYMERS}

Poly(vinylidene fluoride) (PVDF or PVF2) and its copolymers are the most widely exploited ferroelectric polymers. These polymers are partly crystalline, with the amorphous phase being inactive. They possess Young's moduli near 1-10 GPa. A large amplitude ( $\sim 200$ $\mathrm{MV} / \mathrm{m}$ ) applied $\mathrm{AC}$ field can induce electrostrictive (nonlinear) strains of nearly $2 \%$. However this level of field is dangerously close to dielectric breakdown, and the dielectric hysteresis (loss, heating) is very large, thus limiting it's use in practical devices. Scheinbeim [Sen, 1984] has investigated the effect of heavily plasticizing ( $\sim 65 \mathrm{wt} . \%)$ ferroelectric polymers hoping to achieve large strains at reasonable applied fields. However, the plasticizer is also amorphous and inactive, resulting in decreased Young's modulus, permittivity and electrostrictive strains. Recently, Zhang [1998] has introduced defects using electron radiation to reduce the dielectric loss dramatically in P(VDF-TrFE) copolymer. This permits $\mathrm{AC}$ switching with a lot less heat generated. As large as $4 \%$ electrostrictive strains can be achieved at low frequency drive fields of amplitudes $\sim 150 \mathrm{~V} / \mu \mathrm{m}$. The elastic modulus of this material is $0.4 \mathrm{GPa}$, and therefore the mechanical energy density is quite large.

As with ceramic ferroelectrics, electrostriction can be considered as the origin of piezoelectricity in ferroelectric polymers [Furukawa, 1990]. A DC bias polarization can either be present via a poling process before use in a device, in which case a remnant polarization persists, or large DC electric field is applied during operation of the material in a device. In the latter case, no remnant polarization is observed when the bias is removed, and corresponds to a ferroelectric possessing a very small hystersis in the polarization-electric field loop. Unlike electrostriction, piezoelectricity is a linear effect. Not only will the material strain when voltage is applied, but a voltage signal will be induced when a stress is applied. This enables them to be used as sensors. Care must be given to not apply too large of applied voltage, mechanical stress, or high temperature for fear of de-poling the material.

\section{ELECTRO-VISCOELEASTIC ELASTOMERS}

Electro-viscoeleastic elastomers represent another family of electroactive polymers. These EAP materials are composites of silicone elastomer and a polar phase. Before crosslinking, in the uncured state, they behave as electro-rheological fluids. An electric field is applied during curing to orient and fix in position the polar phase in the elastomeric matrix. These materials then remain in the "solid" state but have a shear modulus (both real and imaginary parts) that changes with applied electric field $(<6 \mathrm{~V} / \mu \mathrm{m})$ [Shiga, 1997]. A stronger magneto-rheological effect can also be introduced in an analogous manner and as much as a $50 \%$ change in the shear modulus can be induced [Davis, 1999]. These materials may be used as alternatives to 
electrorheological fluids for active damping applications. To obtain precision control of robotic arms, with a closed-loop system, active damping is necessary.

\section{DEVELOPMENT OF EAP FOR SPACE APPLICATIONS}

Since 1995, under the author's lead, planetary applications using EAP have been explored while improving the understanding, practicality and robustness of these materials. EAP materials are being sought as a substitute for conventional actuators, such as motors, gears, bearings, screws, etc. [Bar-Cohen, et al, 1999b]. Generally, space applications are the most demanding in terms of operating conditions, robustness and durability offering an enormous challenge and great potential for these materials. Under this NASA funded effort, ESSP and IPMC were used to produce longitudinal and bending actuators, where a dust-wiper, gripper and robotic arm were demonstrated. The development of a dust-wiper (Figure 10) was considered for the Nanorover's optical/IR

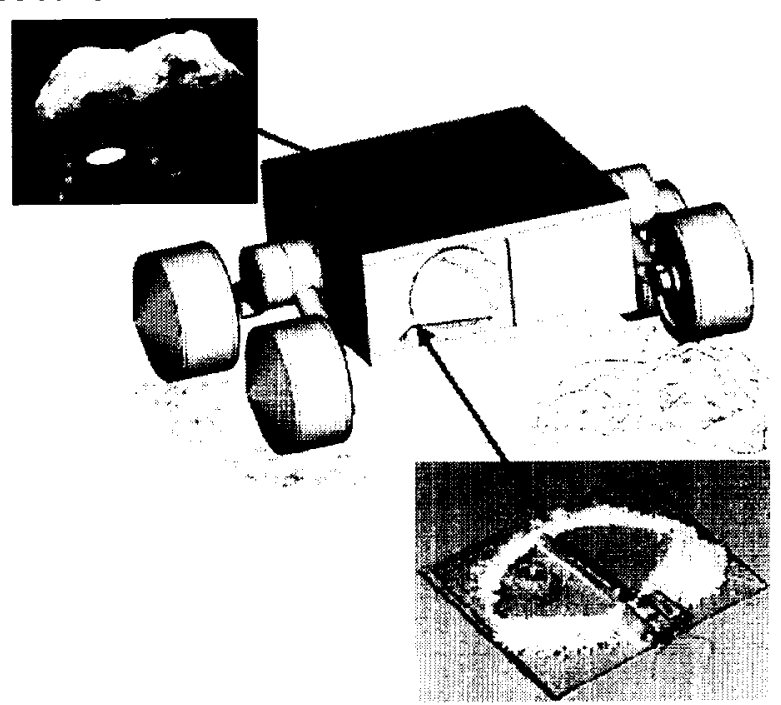

FIGURE 10: Schematic view of the EAP dust-wiper on the MUSES-CN's Nanorover (right) and a photograph of a prototype EAP dust-wiper (left). window, which is part of the MUSES-CN mission. The MUSES-CN is a joint NASA and Japanese Space Agency mission scheduled for launch in January 2002, from Kagoshima, Japan, to explore the surface of a small near-Earth asteroid. The use of IPMC was investigated jointly with NASA LaRC from the USA and with Osaka National Research Institute and Kobe University from Japan. The team used perfluorocarboxylate-gold composite with two types of cations, tetra-n-butylammonium and lithium. The IPMC was used as the actuator of a unique $\sim 100-\mathrm{mg}$ blade with fiberglass brush was developed by ESLI (San Diego, CA). This blade is subjected to high voltage $(\sim 1.5-\mathrm{V})$ to repel dust and thus augmenting the brushing mechanism provided by the blade. Unfortunately, the critical issues that were identified with regards to the application of IPMC is hampering the application from further consideration for inclusion in this planetary mission.

Another application of EAP actuators, having even lower technology readiness, that was considered is the development of a miniature robotic arm (Figure 11). An ESSP actuator was used to lift and drop the arm, whereas a 4-finger IPMC gripper was used to grab rocks and other objects. The EAP fingers operate much like a human hand when grabbing the rock as shown in Figure 12.

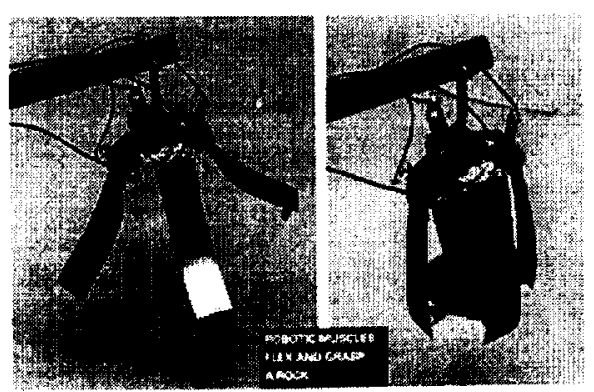

FIGURE 11: 4-finger EAP gripper lifting a rock much like a human hand.
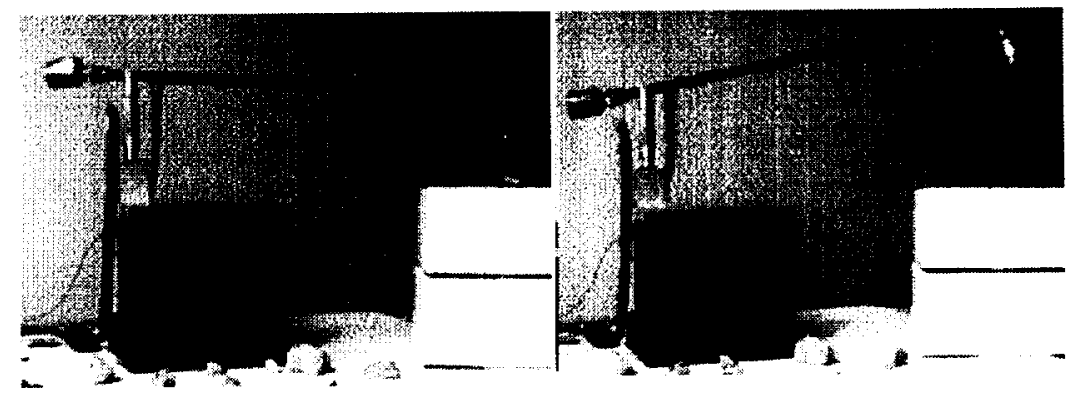

FIGURE 12: A miniature robotic arm using EAP actuators to provide the lifting/dropping of the arm and manipulate the gripper fingers. 


\section{CONCLUDING REMARKS}

In recent years, electroactive polymers have emerged with great potential to enabling unique mechanisms that can emulate biological systems. A series of materials were reported to induce large longitudinal and bending actuation. Efforts to apply such materials to space applications revealed critical challenges that cannot be address with current technology. Much more research and development work still needs to be done before EAP will become the actuators of choice. The development of an effective infrastructure for this field is critical to the commercial availability of robust EAP actuators for practical applications. The challenges are enormous, but the recent international trend towards more cooperation and greater visibility to the field as well as the surge in funding and research is offering great hope for the future of these exciting materials. The potential to operate biologically inspired mechanisms driven by EAP, i.e., artificial muscles, is offering exciting applications that are currently considered science fiction. To highlight this potential, the has challenged the EAP science and engineering community to develop a robotic hand actuated by EAP that would win an arm-wrestling match against human opponent. Progress towards this goal will lead to great benefits to mankind particularly in the medical area including effective prosthetics.

\section{ACKNOWLEDGEMENT}

The research at Jet Propulsion Laboratory (JPL), California Institute of Technology, was carried out under a contract with National Aeronautics Space Agency (NASA). The author would like to thank his LoMMAs team members Dr. S. Leary, JPL, Dr. J. Harrison and Dr. J. Smith, NASA LaRC, Prof. R. Claus, VT, Prof. C. Mavroidis, and C. Pfeiffer, Rutgers U., as well as Dr. T. Knowles, ESLI. He would like to thank Prof. P. Calvert, University of Arizona, for his material on biological muscles and to Prof. M. Shahinpoor for providing the IPMC material that was used in the early phases of the LoMMAs task. For assisting with the development of the EAP dustwiper, the author would like also to express special thanks to Dr. K. Oguro, Osaka National Research Institute, Osaka, Japan for contributing the most recent IPMC materials and to Prof. S. Tadokoro, Kobe University, Kobe, Japan for analytical modeling effort.

\section{REFERENCE}

Alexander R. M., Elastic Mechanisms in Animal Movement, The Cambridge University Press: Cambridge, 1988.

Bar-Cohen Y., T. Xue, M. Shahinpoor, J. O. Simpson, and J. Smith, "Flexible, low-mass robotic arm actuated by EAP," Proceedings of the SPIE International Smart Materials \& Structures Conference," SPIE Paper No. 3329-07, San Diego, CA, 1-6 (March 1998).

Bar-Cohen, Y., (Ed.), Proceedings of the Electroactive Polymer Actuators and Devices, Smart Structures and Materials 1999, Volume 3669, pp. 1-414, (1999a).

Bar-Cohen, Y., K. Oguro, S. Tadokoro, S. Leary, J. Harrison and J. Smith "Challenges to the Transition of IPMC Artificial Muscle Actuators to Practical Application," Proceedings of the Fall MRS Meeting, (1999b).

Baughman, R., "Carbon Nanotube Artificial Muscles and Sensors," Proceedings of the MRS Symposium on Electroactive Polymers, Symposium FF, Bar-Cohen, Furukawa, Scheinbeim and Zhang (Eds.), Boston, MA, Nov. 1999, paper FF 1.3.

Block, H. and Kelly, J. P., 1988, "Electro-Rhehology", Journal of Physics, D: Applied Physics, Vol. 21, pp. 1661.

Calvert P., J. O'Kelly, C. Souvignier, "Solid Freeform Fabrication of Organic-Inorganic Hybrid Materials" Mater. Sci. Eng. C 6, 167-174 (1998)

Davis, L.C., "Model of magnetorheological elastomers," J. Appl. Phys, Vol.85, No.6 (1999), pp.3348-3351.

Eguchi M., Phil. Mag., Vol. 49, (1925) 
Firoozbakhsh, K., and M. Shahinpoor, "Mathematical Modeling of Ionic Interactions and Deformation," Proc. SPIE Smart Materials and Structures Conf., March 3-5, 1998, San Diego, CA, Publication No. SPIE 3323-66, (1998)

Full, R.J., and Tu, M.S., "Mechanics of six-legged runners.” J. Exp. Biol. Vol.148, pp. 129-146 (1990).

Furukawa, T. and Seo, N., "Electrostriction as the origin of piezoelectricity in ferroelectric polymers," Jpn. J. Appl. Phys, Vol. 29, No. 4 (1990), pp. 675-680.

Heitner-Wirguin, C. "Recent advances in perfluorinated ionomer membranes: Structure, properties and applications," Journal of Membrane Science, V 120, No. 1, pp. 1-33, 1996.

Kuhn W., B. Hargitay, A. Katchalsky, and H. Eisenburg, "Reversible dilatation and contraction by changing the state of ionization of high-polymer acid networks," Nature, Vol. 165 (1950), pp. 514-516.

Li F. K., W. Zhu, X. Zhang, C. T. Zhao, and M. Xu, "Shape memory effect of ethylene-vinyl acetate copolymers," J. Appl. Polym. Sci., Vol. 71, No. 7 (1999), pp. 1063-1070.

Nemat-Naser S., and J. Y. Li, "Electromechanical Response of Ionic Polymer-Metal Composites," accepted for publication in J. Applied Physics.

Oguro K., N. Fujiwara, K. Asaka, K. Onishi, and S. Sewa, "Polymer electrolyte actuator with gold electrodes," Proceedings of SPIE's $6^{\text {th }}$ Annual International Symposium on Smart Structures and Materials, Newport Beach, CA, paper 3669-39, 1-5 March, 1999

Oguro, K., Y. Kawami and H. Takenaka, "Bending of an Ion-Conducting Polymer FilmElectrode Composite by an Electric Stimulus at Low Voltage," Trans. Journal of Micromachine Society, Vol. 5, (1992) pp. 27-30.

Otero T. F., H. Grande, J. Rodriguez, "A new model for electrochemical oxidation of polypyrrole under conformational relaxation control," J. Electroanal. Chem., Vol. 394 (1995), pp. 211-216.

Perline R. E., R. D. Kornbluh, and J. P. Joseph, "Electrostriction of polymer dielectrics with compliant electrodes as a means of actuation," Sensor Actuat. A, Vol. 64 (1998), p.77-85.

Sadeghipour, K., R. Salomon, and S. Neogi, "Development of a Novel Electrochemically Active Membrane and 'Smart' Material Based Vibration Sensor/Damper," Smart Materials and Structures, (1992) 172-179.

Schulte, H.F., "The Characteristics of the McKibben Artificial Muscle" Applications of External Power in Prosthetics and Orthotics, Appendix H, Publication 874, National Academy of Sciences, Washington, pp. 94-115, (1961).

Sen, A., Scheinbeim, J.I., and Newman, B.A., "The effect of platicizer on the polarization of poly(vinylidene fluoride) films," J. Appl. Phys., Vol.56, No.9, pp.2433-2439 (1984).

Shahinpoor, M., "Conceptual Design, Kinematics and Dynamics of Swimming Robotic Structures using Ionic Polymeric Gel Muscles," Smart Materials and Structures, Vol. 1, No. 1 (1992) pp. 91-94.

Shiga, T. "Deformation and viscoelastic behaviour of polymer gels in electric fields," $A d v$. Polym. Sci., Vol. 134, pp. 131-163 (1997).

Sokolowski, W. M., A. B. Chmielewski, S. Hayashi and T. Yamada, "Cold Hibernated Elastic Memory (CHEM) Self-Deployable Structures," Proceedings of the Electroactive Polymer Actuators and Devices, Smart Structures and Materials 1999, Volume 3669, Y. Bar-Cohen (Ed.), pp. 179-185, (1999).

Steinberg I. Z., A. Oplatka, and A. Katchalsky, "Mechanochemical engines," Nature, vol. 210, (1966) pp.568-571.

Sussman M. V., "Mechanochemical availability," Nature, Vol. 256 (1975), pp. 195-198.

Tanaka T., I. Nishio, S.T. Sun, and S. U. Nishio, "Collapse of gels in an electric field," Science, vol. 218, pp. 467-469 (1982).

Tobushi H., S. Hayashi, S. Kojima, "Mechanical properties of shape memory polymer of polyurethane series," JSME Int. J., Ser. I. Vol. 35, No 3 (1992), pp.296-302.

van der Veen G. and W. Prins, Phys. Sci., Vol. 230 (1971), pp. 70. 
Yoshiko A., A, Mochizuki, T. Kawashima, S. Tamashita, K. Asaka and K. Oguro, "Effect on Bending Behavior of Counter Cation Species in Perfluorinated Sulfonate MembranePlatinum Composite," Polymers for Advanced Technologies, Vol. 9 (1998), pp. 520-526.

Zhang Q. M ., V. Bharti, and X. Zhao, "Giant electrostriction and relaxor ferroelectric behavior in electron-irradiated poly(vinylidene fluoride-trifluorethylene) copolymer," Science, Vol. 280, pp.2101-2104 (1998).

Zrinyi M., L. Barsi, D. Szabo, and H. G. Kilian, "Direct observation of abrupt shape transition in ferrogels induced by nonuniform magnetic field," J. Chem. Phys., Vol. 106, No. 13 (1997), pp. 5685-5692. 